\title{
Automatic Compensation of Nonlinear Distortions
}

\author{
Tamás B. Bakó ${ }^{1}$, Tamás Dabóczi ${ }^{1}$, Member IEEE and Barry A. Bell ${ }^{2}$, Fellow IEEE \\ ${ }^{1}$ Department of Measurement and Information Systems \\ Budapest University of Technology and Economics, H-1521 Budapest, Hungary \\ Phone: +36 1 463-2057, Fax: +36 1463-4112, email: \{bako, daboczi\}@mit.bme.hu \\ ${ }^{2}$ Electricity Division, National Institute of Standards and Technology, Gaithersburg, MD 20899 USA. \\ email: bell@eeel.nist.gov
}

\begin{abstract}
When a signal passes a system having a static, nonlinear transfer function, the output signal will be distorted. In addition to it, the detected output signal is usually contaminated by noise, due to the noisy environment. If the distortion level of the signal is not acceptable, we have to compensate it. This should be done carefully, because an improper compensation function can extremely amplify the noise. Our aim is to find a compensation function in the knowledge of the original nonlinear transfer function, which can keep both the distortion and the noise level low. In this paper we will show the further developments of our method, which can estimate the optimal level of noise suppression of the compensation function, based on Tikhonov's regularization technique. We will show a method that can find the optimal value of the regularization parameter.
\end{abstract}

$\underline{\text { Keywords }}$ - Nonlinear distortion, nonlinear compensation, regularization, regularization parameter, sound-restoration, restoration of old movie-films

\section{INTRODUCTION}

\section{A. Preliminaries}

Signals can be distorted due to nonlinearity of devices. If the distortion is not acceptable, we have to compensate it. The method of the compensation is not straightforward, when the detected, distorted signal is contaminated by noise. If we use a wrong compensation function, this noise can be extremely amplified and the estimate about the original signal could be completely unusable.

A practical example is the distortion of audio signals. In the case of tape recordings and movie films, the recorded signals could be distorted due to the strong, static, nonlinear behavior of the carrier. The principal causes of nonlinearities in soundrecordings are saturation in magnetic recordings, tracing distortions, groove deformations in gramophone-records and the inherent nonlinearity of variable density optical soundtracks. These distortions disturb the artistic enjoyment and sometimes make the sound-recording incomprehensible.

The first trial to reduce nonlinear distortions in audio recordings was by S. A. White [1]. He used a histogram equalization technique. An iterative method was shown by Preis and Pohlclopek [2]. Troughton and Godsill [3] proposed another iterative method, which is based on a nonlinear autoregressive model. The parameters of the model were computed by a Bayesian approach. Other techniques were also pre- sented as compensation of loudspeaker nonlinearities [4] and compensation of nonlinearities of cathode-ray tubes [5].

The problem of these techniques is that they are mainly iterative techniques and require high computational time. Many of these algorithms use signal prefiltering. In this case the effects of noise are negligible. However, in certain cases, we do not have access to the input of the system and we cannot prefilter the signal. Our aim was to develop a technique that is a non-iterative postfiltering method and can handle the problems produced by the noise. We developed a technique, which was presented in [6]. This technique is based on Tikhonov's regularization method and provides good speed during signal restoration. However, in that time, we could not compute the exact value of the regularization parameters. We could only find an approximation with a subjective decision by listening to some estimated audio signal, computed with different regularization value. This paper describes how we overcame these difficulties and made the restoration procedure completely automatic.

\section{B. Novelty}

In Section II, we will present the mathematical background of using the Tikhonov regularization. In Section III, we will show a novel method that can compute the parameters of the compensation characteristics, knowing the static, nonlinear distortion function, the probability density function of the original signal and the probability density function of the noise. For cases where we do not know any information about the original signal and we do not know the density function of it, we developed a method that can produce an acceptable estimate from the output signal. This density function estimation algorithm will also be shown in Section III. In Section IV, a simulation example will be presented. Conclusions will be given in Section V.

\section{MODELS}

The model of the signal restoration process can be seen in Figure1. The estimated signal can be described as

$$
\hat{x}=K(N(x)+n)=K(o+n),
$$


where $x, \hat{x}, o$ and $n$ refer to the original, the estimated, the distorted and the noise signal respectively. $N($ ) is the static, nonlinear transfer characteristics of the distorting device, and $K($ ) is our compensation function.

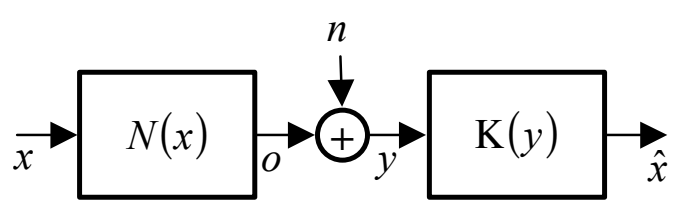

Figure 1 Model of signal restoration.

The evaluation of this equation is difficult, due to the nonlinear functions. In the case of small signal variations, the nonlinear functions can be well described with the first elements of their Taylor polynomials:

$$
\begin{aligned}
& N\left(x_{0}+\Delta x\right)=N\left(x_{0}\right)+\left.\frac{d N(x)}{d x}\right|_{x=x_{0}} \cdot \Delta x, \\
& K\left(y_{0}+\Delta y\right)=K\left(y_{0}\right)+\left.\frac{d K(y)}{d y}\right|_{y=y_{0}} \cdot \Delta y .
\end{aligned}
$$

The signal model for small variations can be seen in Figure 2.

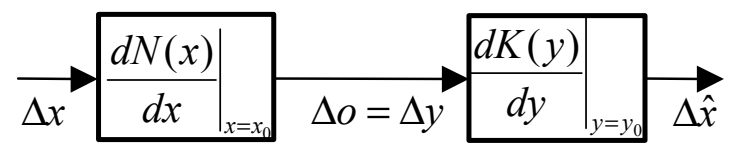

Figure 2 Signal model for small signal changes.

In this case the noise is not present directly in this model, but affects the amplification of the compensation block. The estimated signal can be computed as

$$
\Delta \hat{x}=\left.\frac{d K(o+n)}{d o}\right|_{o=o_{0}} \cdot \Delta y=\left.\frac{d K(y)}{d y}\right|_{y=y_{0}} \cdot \Delta y .
$$

For compensation, the reciprocal of $\frac{d N(x)}{d x}$ seems to be obvious. This is the solution of the output error criterion:

$$
\operatorname{Cost}=\min _{\Delta \hat{x}}(\|\Delta y-\Delta \hat{y}\|) .
$$

However, it was shown in [6] that in this case the noise can be extremely amplified. For the solution of this problem, we propose to use one form of Tikhonov-regularization:

$$
\operatorname{Cost}=\min _{\Delta \hat{x}}(\|\Delta y-\Delta \hat{y}\|+\lambda\|\Delta \hat{x}\|)
$$

where $\lambda$ denotes the Tikhonov regularization parameter, which makes a trade-off between bias and noise-level. The solution of this criterion for $\frac{d K(y)}{d y}$ is:

$$
\frac{\Delta \hat{x}}{\Delta y}=\left.\frac{d K(y)}{d y}\right|_{y=y_{0}}=\frac{\left.\frac{d N(\hat{x})}{d \hat{x}}\right|_{\hat{x}=\hat{x}_{0}}}{\left(\left.\frac{d N(\hat{x})}{d \hat{x}}\right|_{\hat{x}=\hat{x}_{0}}\right)^{2}+\lambda}
$$

\section{FINDING THE EXACT VALUE OF THE REGULARIZATION PARAMETER}

In [6], the optimal value of the regularization parameter was found with a subjective decision. We computed several compensation functions with different regularization parameters and we chose the best sound record estimate, which had the "clearest" voice. This decision can be done in the case of an audio file; however, we cannot use this technique in the case of a distorted measurement file. In this case we have to use an automatic method to omit human interaction.

The optimal value of the regularization parameter, $\lambda$, depends on the input signal, on the noise and on the shape of the original nonlinear function. If the input signal, $x$, is constant, and if the probability density function of the output noise, $P_{n}(v)$, is known, the norm of the difference between the original signal and the estimate can be written in the following form:

$$
\begin{aligned}
& \underset{n}{E}\{e(x, \lambda)\}=E\{\|\hat{x}(\lambda)-x\|\}= \\
& =E\{\|\mathrm{~K}(y, \lambda)-x\|\}= \\
& =\int_{-\infty}^{\infty} P_{n}(v) \cdot\|\mathrm{K}((N(x)+v), \lambda)-x\| d v,
\end{aligned}
$$

where $e(x, \lambda)$ refers to the difference of the original and the estimated signal. Now, the expected error value can be computed for all given $x$ values. If the probability density function, $P_{x}(\chi)$, of $x$ is known, the expected error after restoration can be written as

$$
\underset{x}{E}\{E\{e(x, \lambda)\}\}=\int_{-\infty}^{\infty} P_{x}(\chi) \cdot E\{e(x, \lambda)\} d \chi
$$

The optimal value of $\lambda$ can be found by minimizing (8). In practice, $P_{n}(v)$ can be estimated from those signal parts where only noise is present. However this method requires 
the knowledge of the probability density function of the original signal, which is often not known.

In this case $P_{x}(\chi)$ can be estimated iteratively from the output signal. As the first step, $P_{x}(\chi)$ is approximated by the probability density function of the observed signal, $P_{o}(o)$. Now, $\lambda_{1}$ and $\hat{x}_{1}$ can be estimated. From $\hat{x}_{1}$, a more exact estimate for $P_{x}(\chi)$ can be obtained. In our experiments, three iterations were enough to estimate a proper $P_{x}(\chi)$, and have a proper $\lambda$ value.

\section{SIMULATION}

To show the capabilities of the proposed algorithms, a multisinusoidal input signal consisting of four sinusoids was used. This signal can be seen in Fig. 4a. Then this signal was distorted by a Gaussian error-function, which is similar to the density function of films:

$$
N(x(t))=0.5 \cdot \operatorname{erf}(x(t)+0.5)-0.5
$$

A Gaussian white noise was added to the signal to achieve a $35 \mathrm{~dB}$ signal-to-noise ratio. The resultant distorted, noisy signal can be seen in Fig. $4 \mathrm{~b}$.

The distortion function is known, but we assume that the probability density-characteristics of the original signal is not. Hence, the optimal inverse characteristics were calculated by the method proposed in Section 3. Here, five iterations were made and the resultant value was compared with the real optimal value, computed from the density-characteristics of the original signal. The resultant $\lambda$ values and the squared sum of the difference of the original and the estimated signal are given in Table I:

Table I: Regularization and error parameters of different regularized characteristics:

\begin{tabular}{|l|l|l|}
\hline \multicolumn{3}{|c|}{ characteristics: } \\
\hline Underregularized & $1 \cdot 10^{-10}$ & 206.087 \\
\hline Optimal & $3.036 \cdot 10^{-4}$ & 6.388 \\
\hline Estimated & $1.272 \cdot 10^{-4}$ & 7.499 \\
\hline Overregularized & $10^{-1}$ & 191.345 \\
\hline
\end{tabular}

The difference between the optimal and estimated error is relatively small.

The regularized inverses for the underregularized, optimal, estimated and overregularized cases can be seen in Fig. 5. Fig. 6 shows the reconstructed signals. The underregularized inverse has large errors, due to the noise amplification. The overregularized inverse has strong distortion. In the case of the optimal and estimated characteristics, both the effects of distortion and noise are small.

The method was tested also on real audio signals and performs well.

\section{CONCLUSIONS}

In this paper a method for nonlinear compensation was described that can provide an optimal output in the case of noisy signals. The method is based on Tikhonov's regularization technique. A novel method was shown, which is able to find automatically the optimal value of the regularization parameter; it does not require human interaction. The required known parameters are the nonlinear distortion function and the probability density functions of the original signal and the noise. Even if the probability density function of the original signal is not known, a good approximation can be computed with an iterative method from the observed output signal, which was also described. The effectiveness of the method was shown on a simulation example.

\section{ACKNOWLEDGEMENT}

The financial support of the Hungarian Scientific Research Fund (OTKA F-034900) and the Research and Developement Fund for Higher Education (FKFP-0098/2001) is acknowledged.

\section{REFERENCES}

[1] Stanley A. White, "Restoration of Nonlinearly Distorted Audio by Histogram Equalization," J. Audio Eng. Soc., vol. 30, No 11, November 1982, pp. 828-832.

[2] D. Preis and H. Polchlopek, "Restoration of Nonlinearly Distorted Magnetic Recordings," J. Audio Eng. Soc., vol. 32, No 1/2, January/February 1984, pp. 26-30.

[3] Paul T. Troughton and Simon J. Godsill, "Restoration of Nonlinearly Distorted Audio using Markov Chain Monte Carlo Methods," Presented at the $104^{\text {th }}$ Convention of the Audio Engineering Society, preprint 4679, Amsterdam, May 1998.

[4] Wolfgang Klippel, "Compensation for Nonlinear Distortion of Horn Loudspeakers by Digital Signal Processing," AES: Journal of the Audio Engineering Society, vol. 44, No 6, June 1996, pp. 470-480.

[5] S. Kerry Wilson and Patrick Delay, "A Method to Improve Cathode ray Oscilloscope Accuracy," IEEE Trans. Instrumentation and Measurement, vol. 43, No 3, June 1994, pp.483-486.

[6] Tamás B. Bakó, Tamás Dabóczi, "Restoration of Nonlinearly Distorted Signals with Regularized Inverse Characteristics," IEEE IMTC 2001, Budapest, May 21-23, 2001, pp. 1565-1570.

[7] Tikhonov, A. N. and Arsenin, V. Y., "Solutions of ill-posed problems," New York, John Wiley \& Sons, Inc. 1977. 
a)

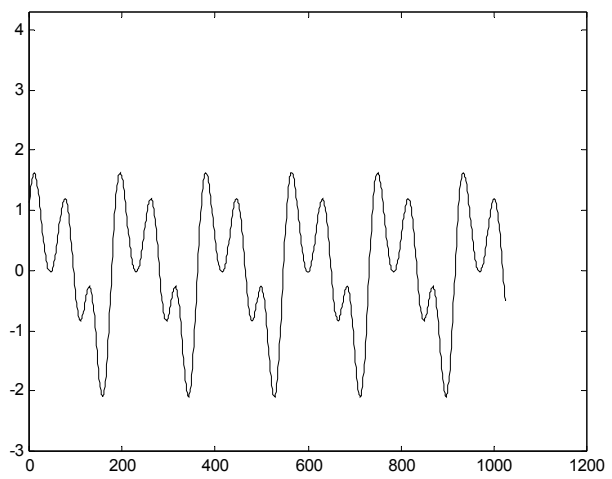

b)

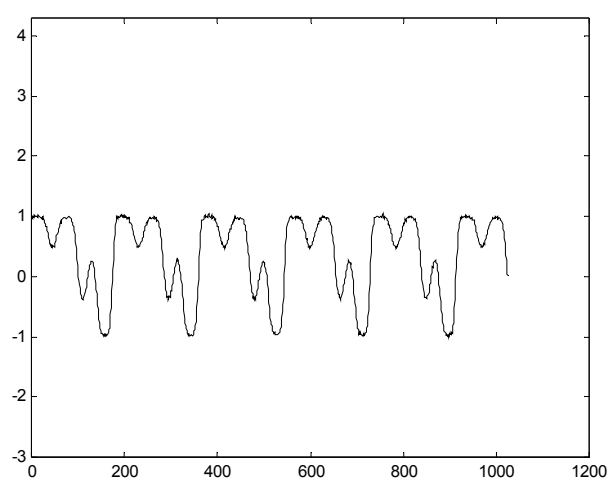

Figure 4: Input signal (a) and the nonlinearly distorted, noisy signal (b).

a)
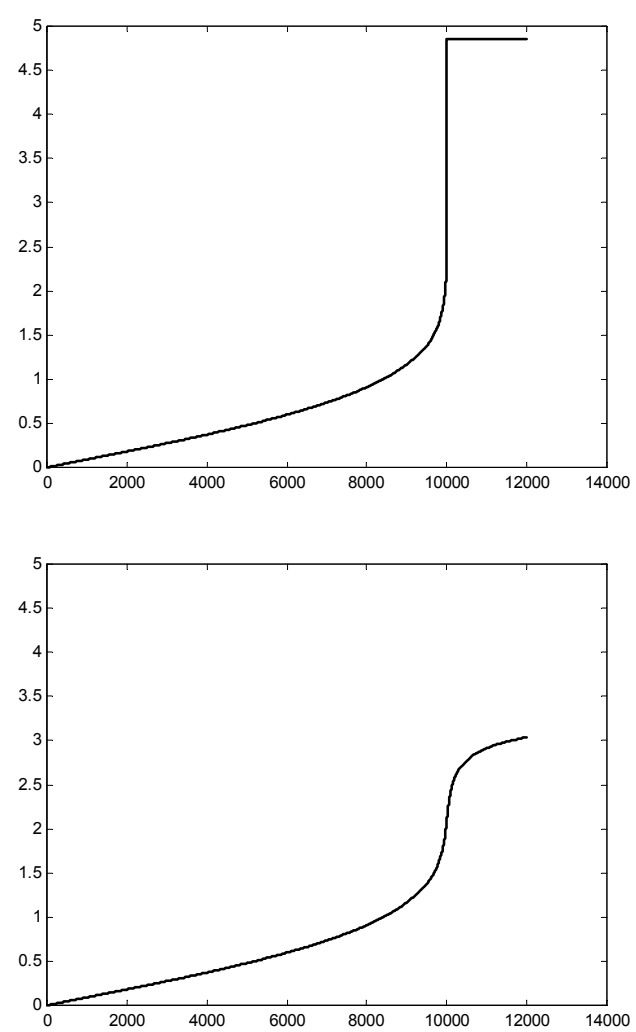

b)

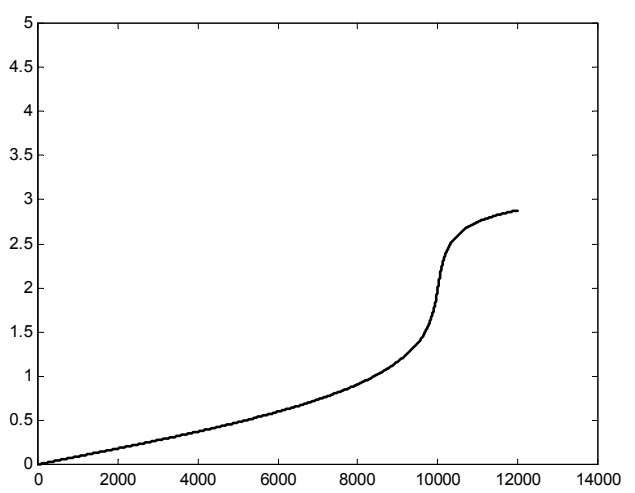

d)

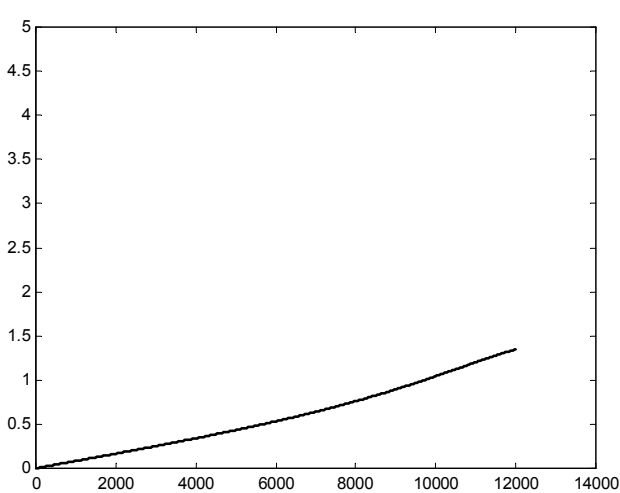

Figure 5: Underregularized (a), optimal regularized (b), estimated regularized (c) and overregularized (d) characteristics. 
a)

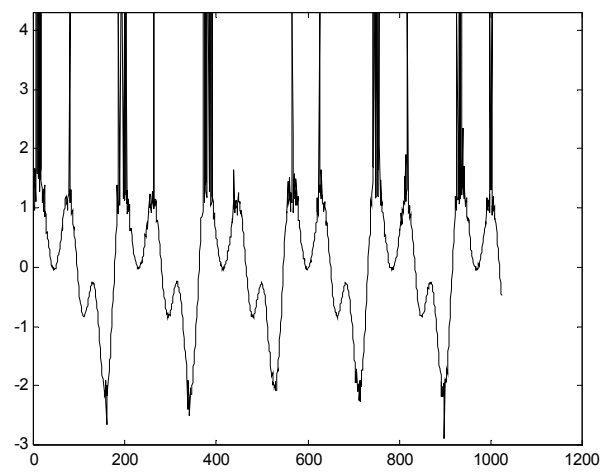

c)

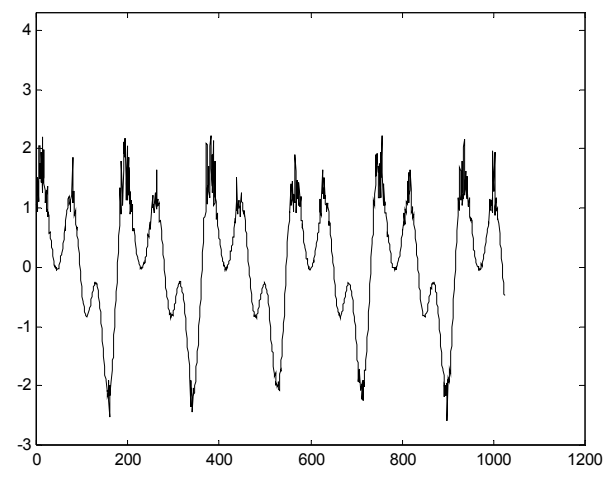

b)

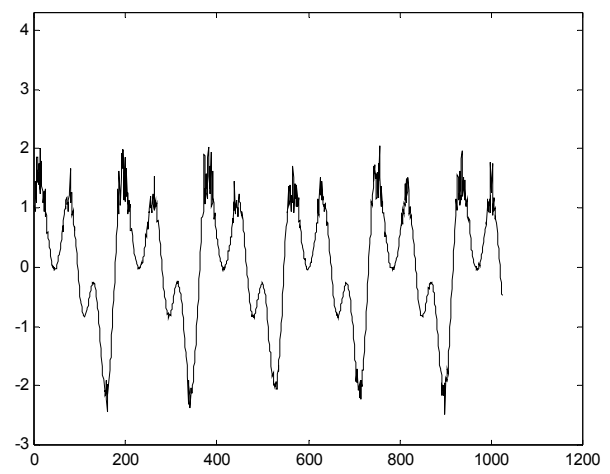

d)

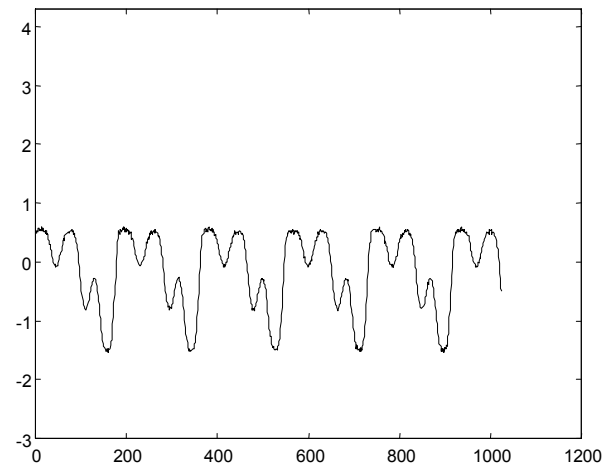

Fig. 6 Reconstructed signals: with underregularized (a), with optimal regularized (b), estimated regularized (c) and overregularized (d) characteristics. 